\title{
Impaired mammary gland T cell population during early lactation in hypoprolactinemic lactation-deficient rats
}

\author{
Juan Pablo Mackern-Oberti ${ }^{1}$, Susana Ruth Valdez ${ }^{1,2}$, Laura María Vargas-Roig ${ }^{1}$ \\ and Graciela Alma Jahn ${ }^{1}$ \\ ${ }^{1}$ Instituto de Medicina y Biología Experimental de Cuyo, CCT Mendoza, CONICET, Mendoza CP5500, Argentina \\ and ${ }^{2}$ Instituto de Ciencias Básicas, Universidad Nacional de Cuyo, Mendoza CP5500, Argentina
}

Correspondence should be addressed to G A Jahn; Email: gjahn@mendoza-conicet.gob.ar JPMackern-Oberti; Email: jpmackern@mendoza-conicet.gob.ar

J P Mackern-Oberti and S R Valdez contributed equally to the experimental design, analysis and writing of this work

\begin{abstract}
Mammary stroma is composed of various cell types, including migratory leukocytes. Although mammary antibody-secreting cells have been extensively studied, reports focusing on mammary $\mathrm{T}$ cells are scarce. It is thought that the recruitment mechanism of leukocytes to the mammary gland (MG) is controlled by pregnancy- and lactation-specific stimuli. But whether prolactin (PRL) modulates the T-cell population in MG is still unknown. Our aim was to study the relationship between PRL levels and T and B cells during early lactation (L2, day 2 post partum) and mid-lactation (L12, day 12 of lactation). In order to investigate whether PRL is associated with homing events to MG, female Sprague Dawley (SD) and SD-derived desmoglein $4^{-/-}$hairless (phenotype with lactation deficit, OFA $h r / h r$ ) rats were killed during estrus, pregnancy, and post partum, and blood, MG, and corpora lutea were obtained to perform fluorescent-activated cell sorting (FACS), real-time PCR, and histological and RIA studies. Serum PRL levels were lower in OFA $h r / h r$ rats than in SD rats during early lactation. MG of OFA $h r / h r$ rats showed less secretory material compared with SD rats. FACS analysis showed lower percentage of

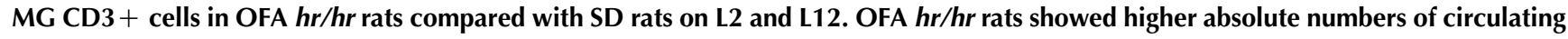
CD3 + cells compared with SD rats on L2 but not on L12. These results show that T-cell population in MG is affected in early lactating OFA $h r / h r$ rats and strongly suggest that serum PRL levels may be involved in the homing events to MG, probably helping antibody-secreting cells and protecting the gland during lactation development.

Reproduction (2013) 146 233-242
\end{abstract}

\section{Introduction}

Immune and endocrine systems interact in a complex network in which hormones may influence immune responses. Several reports have shown that prolactin (PRL) can stimulate T cells, B cells, natural killer cells, macrophages, neutrophils, and dendritic cells (Kooijman et al. 1996, Dogusan et al. 2001, Matera et al. 2001). Leukocytes produce PRL as an autocrine or a paracrine factor influencing cytokine production (Dimitrov et al. 2004). The fact that PRL expression in T cells is regulated by interleukin (IL) 2, IL4, and IL1 $\beta$ (Gerlo et al. 2005) supports the notion of the existence of bidirectional dynamic modulation between immune and endocrine systems.

Although PRL-deficient animals show normal development and function of T- and B-cell populations (Horseman et al. 1997, Bouchard et al. 1999), hypophysectomized animals with low PRL levels show impaired B- and T-cell-mediated immune responses, which are restored by PRL injections (Morikawa et al. 1993, 1994). All these data give us an idea that PRL could modulate immune cells under an activation-like condition. Patients with autoimmune diseases, such as rheumatoid arthritis and systemic lupus erythematosus (SLE), show a high prevalence of hyperprolactinemia, suggesting that this hormone is to some extent involved in autoimmunity (Nagafuchi et al. 1999). Furthermore, PRL exacerbates SLE in F1 NZB/NZW mice (Elbourne et al. 1998), and it increases the survival of autoreactive B cells in susceptible strains (Peeva et al. 2004).

During pregnancy and lactation, the mammary gland (MG) expands its vasculature and epithelium (Yasugi et al. 1989, Watson et al. 2011). It has been known since the 1970s that plasma cells from gut-associated lymphoid tissue are recruited to MG expressing specific adhesion molecules such as CD44 and CD62L and produce mainly IgA to be secreted to milk, transferring immune protection to neonates (Roux et al. 1977, Weisz-Carrington et al. 1977). Mammary IgA-secreting 
cell numbers increase during pregnancy in parallel with mammary lobulo-alveolar compartment expansion, reaching maximal levels post partum (Parmely \& Manning 1983, Tanneau et al. 1999, van der Feltz et al. 2001). Lymphocyte homing is an extremely highly regulated mechanism modulated by chemokines, cytokines, integrins, and addressins (Kunkel \& Butcher 2002).

In contrast, the role of T cells recruited to the MG has not yet been studied in detail. It is proposed that recruited MG T cells may be involved in local antibody secretion, regulation, and protection against infection and thereby in the maintenance of local immune homeostasis (Chabaudie et al. 1993, Tanneau et al. 1999). Although the main roles of PRL in MG development and differentiation during pregnancy and lactation are well characterized, and its role in MG T-cell modulation is poorly understood. As several studies have demonstrated that PRL modulates immune cells, including stimulation of cell adhesion (Montes de Oca et al. 2005), it is likely that PRL exerts some function in T-cell homing to MG during early lactation when the circulating PRL level is high.

The aim of this study was to explore the role of PRL in mammary T-cell population during early lactation using the lactation-deficient OFA $h r / h r$ rats as a model (Valdez et al. 2007, 2012). This strain of rats has a large deletion in the desmoglein 4 gene (Bazzi et al. 2004) and shows a partial blockade of the sucking stimulus resulting in low circulating PRL level only during early, and not in mid- or late lactation that leads to a severe impairment in lactation (Valdez et al. 2007, 2012). This model may provide an optimal in vivo condition for studying the association between PRL levels and T-cell homing during early lactation. We observed that numbers of T cells are decreased in MG of OFA rats and they are increased in peripheral blood during early lactation under low circulating PRL levels, while during mid-lactation, the numbers of $T$ cells continue to be low in MG but are in the normal range in circulation. These results suggest that PRL may modulate, at least in part, T-cell homing events to MG.

\section{Materials and methods Animals}

Adult female and male Sprague Dawley (SD) and OFA $h r / h r$ rats (originally purchased from Iffa Credo, Oncins, France, and denominated IFL Nu at that time) bred in our laboratory were used. The rats used aged between 3 and 4 months, weighing 200-230 g. Animals were kept in a light-controlled (lights on 0600-2000 h) and temperature-controlled (22-24 $\left.{ }^{\circ} \mathrm{C}\right)$ room. Rat chow (Cargill, Córdoba, Argentina) and tap water were available ad libitum. For the experiments, vaginal smears were collected daily from young (3-4 months old) female rats and only those showing regular 4-day cycles were used. To identify estrous cycle and to familiarize rats with handling, vaginal smears were collected using a plastic pipette with $\mathrm{NaCl} 0.9 \%$ during 8 consecutive days at $9-10 \mathrm{~h}$ and placed on glass slides. The smears were immediately analyzed under a microscope and the identification of the phases was done according to the predominant cell type, i.e. proestrus ( $\mathrm{P}$, smear predominated by nucleated epithelial cells), estrus ( $E$, smear predominated by cornified epithelial cells), and first or second diestrus days (D1, D2, smear full of leucocytes). Rats with two consecutive normal estrous cycles were selected for the study (Marcondes et al. 2002).

Rats were made pregnant by caging with a fertile male on the night of proestrus. The presence of sperm on the vaginal smear the following morning indicated day 0 of pregnancy. Two or 3 days before delivery, the rats were caged individually. The day and approximate hour of delivery and the size and weight of the litters were recorded. On day 1 of lactation, the number of pups in each litter was standardized to eight. Animal maintenance and handling were performed according to the $\mathrm{NIH}$ guide for the Care and Use of Laboratory Animals (NIH publication $\mathrm{N}^{\circ}$ 86-23, revised 1985 and 1991) and the UK requirements for ethics of animal experimentation (Animals Scientific Procedures, Act 1986). All the experimental procedures were approved by the Committee of ethics in animal experimentation of the Medical School, Universidad Nacional de Cuyo, Argentina. For the determination of hormones and leukocyte number in virgin, pregnant, and early lactating rats, groups of 8-13 SD rats and 8-12 OFA $\mathrm{hr} / \mathrm{hr}$ rats in estrus (virgins), at the end of pregnancy (days 19 and 21 of gestation, G19 and G21 respectively), and during early lactation (day 2 of lactation, L2) and mid-lactation in some experiments (day 12 of lactation, L12) were bled between 0830 and $1000 \mathrm{~h}(0.5-1 \mathrm{ml})$ from the tail vein under light ether anesthesia. Blood was collected in tubes with EDTA in order to evaluate white blood cells using a Neubauer chamber, a hematology analyzer (ADVIA 120, Bayer), or by flow cytometry. Other groups consisting between five and seven SD and OFA $h r / h r$ rats on L2 were killed by decapitation between 0830 and $1000 \mathrm{~h}$, trunk blood was collected, and serum was separated by centrifugation and stored at $-20^{\circ} \mathrm{C}$ until used. The inguinal MG and corpus luteum $(\mathrm{CL})$ were removed, snap frozen in liquid nitrogen, and stored at $-70^{\circ} \mathrm{C}$ until they were analyzed.

\section{PRL and GH determination}

Serum PRL and GH levels were measured by double-antibody RIA using materials generously provided by Dr Parlow and the NHPP (National Hormone and Pituitary Program, HarborUCLA Medical Center, Torrance, CA, USA). The hormones were radio-iodinated using the Chloramine $T$ method and purified by passage through Sephadex G75. The results were expressed in terms of the rat PRL RP-3 or rat GH RP-2 standard preparations. Assay sensitivity was $0.5 \mu \mathrm{g} / \mathrm{l}$ serum for PRL and $\mathrm{GH}$ and the inter- and intra-assay coefficients of variation were $<10 \%$.

\section{Flow cytometry}

Blood samples were obtained from the tail vein. Total cell numbers were measured by counting cells in a Neubauer 
chamber in Turk's solution and with a hematology analyzer (ADVIA 120, Bayer). To ensure that the hematology analyzer provides adequate values for rat blood cells, we also did a morphological inspection and differential counting of blood cells on blood smears (stained with May Grünwald Giemsa) under light microscope. After blood collection, groups of animals on day 2 (L2) or 12 (L12) of lactation were killed by decapitation. Inguinal MG and CL (only on L2) were rapidly dissected and minced under sterile conditions into 1-2 mm fragments and subjected to enzymatic digestion using type IV collagenase $(200 \mathrm{U} / \mathrm{ml})$ and trypsin $(0.25 \%)$ in DMEM/F12 at $37^{\circ} \mathrm{C}$. Local lymph nodes were carefully separated from MG. After incubating for $1 \mathrm{~h}$, cells were dispersed and washed through a $200 \mu \mathrm{m}$ nylon cell strainer. Resident leukocyte populations from peripheral blood, $\mathrm{MG}$, and $\mathrm{CL}$ were evaluated by fluorescent-activated cell sorting (FACS). The following antibodies were used to identify different populations: PE-Cy5-conjugated anti-rat CD45 (clone OX-1; isotype mouse IgG1 k) (BD Biosciences, San Jose, USA); FITCconjugated anti-rat CD45RA/B (clone OX-33; isotype mouse IgG1) (Cedarlane Laboratories, Burlington, ON, Canada), FITCconjugated anti-rat CD3 (clone IF4; isotype mouse IgM) (Cedarlane Laboratories), PE-conjugated anti-rat CD11b (clone WT.5; isotype mouse IgA) (BD-Pharmingen, San Diego, CA, USA), mouse IgG1 anti-rat/human/porcine/rabbit/equine PRL Receptor clone U5 (generously provided by Dr Jean Djiane, Unitéd' Endocrinologie Moleculaire, INRA, Jouy en Josas, France, and Dr Paul A Kelly, INSERM U344, Paris, France), and mouse anti-rabbit PE/FITC (BD Biosciences). Cells were stained according to standard protocols (Holmes et al. 2001). Flow cytometry was performed on a BD FACS Calibur (BD Biosciences), between 20000 and 50000 events were acquired for each sample, and data were analyzed with WinMDI software (Scripps Research Institute, La Jolla, CA, USA).

\section{Histopathology and immunohistochemistry}

After killing, portions of MG and CL were harvested and fixed in buffered formaldehyde and processed for paraffin embedding wax. Five-micrometer sections were cut in a Reichert-Jung $\mathrm{Hn} 40$ microtome and stained with hematoxylin-eosin. Each evaluated section was separated from the next by $10 \mu \mathrm{m}$. Slides were analyzed under a light microscope to evaluate the degree of mammary development. From each animal, five sections of every gland were analyzed. The degree of mammary development was evaluated taking as parameters the relative proportions of lobulo-alveolar tissue and fat pad and the accumulation of secretory material on the alveoli, considering the degree of distension of the alveoli.

For immunohistochemistry, CD3 antigen unmasking was carried out in $0.01 \mathrm{M}$ citrate buffer $\left(\mathrm{pH} \mathrm{6.0)}\right.$ at $100{ }^{\circ} \mathrm{C}$ for $25 \mathrm{~min}$. Sections were incubated with the primary antibody mouse anti-rat CD3 (clone G4.18; mouse isotype IgG3, k) (BD, Pharmingen, USA) (generously provided by Dr Virginia Rivero, $\mathrm{CIBICI}-\mathrm{CONICET}$, Argentina) overnight at $4{ }^{\circ} \mathrm{C}$ in humidity chamber at 1:200 dilution. As a detection method, we used as secondary antibody biotinylated rabbit anti-mouse IgG and avidin-biotin ABC complex (VECTASTAIN Elite ABC System, Vector, Burlingame, CA, USA). Diaminobenzidine (Vector) was used as a chromogen substrate. Slides were lightly counterstained with hematoxylin and observed with an Eclipse E400 microscope (Nikon, Japan). The immunostaining was evaluated according to the percentage of positive lymphocytes. Positive control using rat lymph node and negative controls were included in the assays.

\section{Magnetic cell isolation}

T- and B-cell isolation from peripheral blood was performed by positive isolation using Dynabeads M-450 Tosyl activated following the manufacturer's instructions. Briefly, anti-rat CD45RA/B (Cedarlane, Laboratories) or anti-rat CD3 (Cedarlane, Laboratories) was coupled to Dynabeads. Red blood cells were eliminated by hypotonic red blood lysis buffer $\left(\mathrm{NH}_{4} \mathrm{Cl}\right.$ $0.17 \mathrm{M} ; \mathrm{K}_{2} \mathrm{CO}_{3} 10 \mathrm{mM}$; and EDTA $0.05 \mathrm{mM}$ ). Total leukocytes were incubated with antibody-coupled beads for $30 \mathrm{~min}$ at $4{ }^{\circ} \mathrm{C}$ with gentle tilting and rotation. The reaction tube was placed on a magnet for $2 \mathrm{~min}$ and the supernatant containing the unbound cells was discarded. The purity of T- and B-cell isolations was about $85 \pm 5 \%$. Cells were washed and lysed in TRIzol reagent (Invitrogen, Life Technologies) to isolate total RNA for real-time PCR assays.

\section{Real-time PCR analysis}

Briefly, total RNA was isolated from peripheral blood-isolated $\mathrm{T}$ and $\mathrm{B}$ cells and MG using TRIzol reagent (Invitrogen, Life Technologies) according to the manufacturer's protocol. RT reactions were performed using $2 \mu \mathrm{g}$ of total mRNA in a $25 \mu \mathrm{l}$ mixture. Total RNAwas first incubated with $0.5 \mu$ goligo(dT) primer (Promega) for $10 \mathrm{~min}$ at $65^{\circ} \mathrm{C}$ and allowed to stand at room temperature for $2 \mathrm{~min}$. Samples were then incubated with $1.25 \mathrm{mM}$ dNTPs (Promega), $10 \cup$ RNase Inhibitor (Boehringer, Mannheim), and $16 \mathrm{U}$ avian myeloblastosis virus reverse transcriptase (Promega) for $1 \mathrm{~h}$ at $42{ }^{\circ} \mathrm{C}$ in reverse transcriptase buffer. The cDNA obtained was subjected to PCR amplification using Eva-Green-based real-time PCR in a Rotor Gene 6000 cycler (Corbett, Qiagen). The following primers were used: CCR1 (sense AAGTACCTTCGGCAGCTGTTTC, antisense ACAGAGAAGAAGGGCAGCCAT); CCR2 (sense AACTGTGTGGTTGACATGCACTT, antisense ACGCAGCAGTGTGTCATTCC); CCR3 (sense ATTTCTTGCTCCCCAGTTGATG, antisense CCATGCAAGTGACTGAGGTGATTA); CCR5 (sense GGCAATGCAGGTGACAGAGA, antisense CCCAACAAAGGCATAGATGACA); CCR7 (sense ACAAGGCCATCAAGGTGATCA, antisense TTGACGCCGATGAAGGCAT); CXCR3 (sense AGGTCAGTGAACGTCAAGTGCTAG, antisense GCAAAAAGAGGAGGCTGTAGAGGA); CXCR5 (sense TGCCTTGCCAGAACTCCTCTTT, antisense GCCTGTGTACCACTCCCACATAA), PRL receptor (PRL-R), long form (sense AAAGTATCTTGTCCAGACTCGCTG, antisense AGCAGTTCTTCAGACTTGCCCTT); and actin (sense CGTGGGCCGCCCTAGGCACCA, antisense TTGGCCTTAGGGTTCAGAGGGG). After performing the reactions, real-time amplification data were gathered using Rotor Gene software (Corbett, Qiagen, Los Angeles, CA, USA). Gene expression was normalized to the housekeeping actin gene to determine the fold change in gene expression between test and control samples by $2^{-\Delta \Delta C t}$ method. 


\section{Statistical analysis}

Statistical analysis was performed using one-way ANOVA or using Student's $t$-test when only two groups were compared with Graph Pad Prism software (Graph Pad Software, San Diego, CA, USA). Differences between means were considered significant at the $P<0.05$ level.

\section{Results}

\section{$P R L$ serum levels and MG development during early lactation}

As MG development is a complex mechanism that occurs during pregnancy and lactation, mainly induced by PRL among other pregnancy hormones, we evaluated PRL levels in OFA $h r / h r$ and SD rats during estrus, late pregnancy, and early and mid-lactation. As previously described (Valdez et al. 2007, 2012), we found that OFA $h r / h r$ rats showed lower levels of PRL compared with SD rats only during early lactation (day 2 of lactation, L2) (Fig. 1A). On the other hand, there was no difference in serum GH concentration between strains of rats at each reproductive stage studied (data not shown). When the MG architecture was studied on L2, and confirming previous results (Valdez et al. 2007), OFA $h r / h r$ rats showed a similar degree of alveolar development compared with SD rats, although the size of the MG tended to be smaller. However, the OFA rats had less secretory material content compared with SD rats (Fig. 1B, C, D and E). Although it is well known that $\mathrm{CL}$ formation and maintenance also depend on PRL secretion, OFA $h r / h r$ rats showed no evidence of any histological alteration compared with controls during early lactation (data not shown). These results suggest that the reduced increase in circulating PRL levels observed in OFA $h r / h r$ rats during early lactation is associated with a decrease in MG development but does not affect $C L$ function, at least during this early stage of lactation.

\section{$B$ - and T-cell populations in peripheral blood}

It is well known that lymphocytes populating MG during pregnancy and lactation migrate mainly from gutassociated lymphoid tissue. Cells need to recirculate through the blood to reach the target tissue. In order to explore whether low levels of PRL alter leukocyte populations, we measured total leukocyte, lymphocyte, and monocyte numbers in peripheral blood of OFA $\mathrm{hr} / \mathrm{hr}$ and SD rats during estrus, late pregnancy, and early lactation. We found no differences between both strains on the different days of pregnancy. However, on L2, we observed that OFA $h r / h r$ rats had higher number of circulating leukocytes compared with SD rats (Fig. 2A). Mononuclear cells (monocytes and lymphocytes) may be responsible for this increase, as they were also elevated (Fig. 2B and C). We also found a slight but significant increase only in total leukocyte number in OFA rats during estrus (Fig. 2A). In contrast, there were no differences between strains with regard to the number of neutrophils at any of the studied stages (Fig. 2D).

Thus, as L2 was the state in which we found increased circulating leukocyte numbers associated with a reduced increase in PRL levels observed in OFA $h r / h r$ rats, we focused our study on this day to characterize leukocyte populations. As can be seen in Fig. 3, we found increased numbers of $\mathrm{CD} 3+\mathrm{T}$ cells as well as CD45RA/B + B cells in OFA $h r / h r$ rats compared with $\mathrm{SD}$ rats, both in absolute numbers and percentage of total leukocytes in peripheral blood (Fig. 3A, B, C and D).

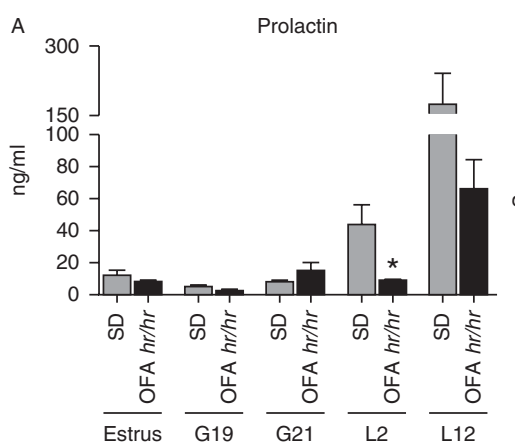

B
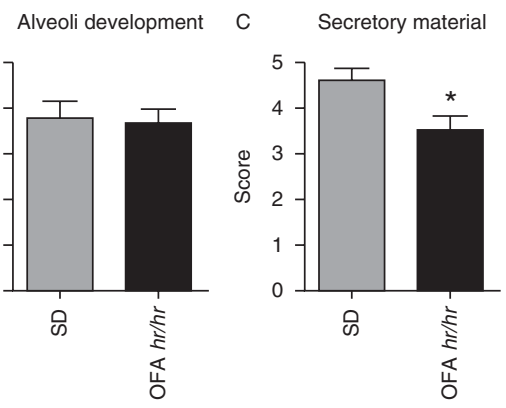

D

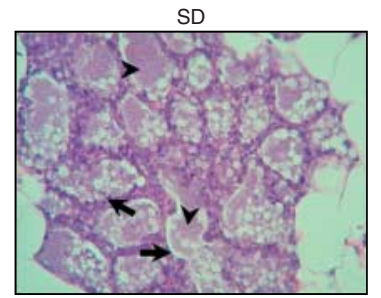

E

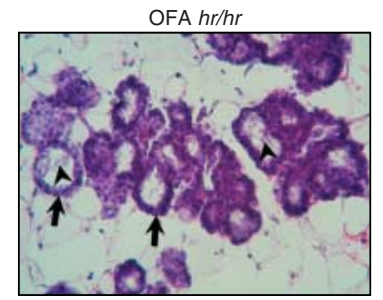

Figure 1 PRL levels and MG development during early lactation. Circulating PRL levels in virgin female (estrus), end of pregnancy (G19 and G21, days 19 and 21 of gestation respectively), and early and mid-lactation (L2 and L12, days 2 and 12 of lactation) from SD (groups of $n=8-13$ ) and OFA $h r / h r(n=8-12)$ rats. (A) Serum PRL levels were measured by RIA. (B, C, D, and E) MG development studied by conventional histology. (B and C) Results of statistical analysis of alveoli development and secretory material (score 0-5). (D and E) Representative microphotographs of hematoxylin-eosinstained sections of MG on L2 from SD and OFA $h r / h r$ rats. Arrows indicate alveoli development and arrowheads indicate presence of secretory material. $\times 20$. Values represent the mean \pm S.E.M. ${ }^{*} P<0.05$; ANOVA test; SD vs OFA $h r / h r$ rats. 

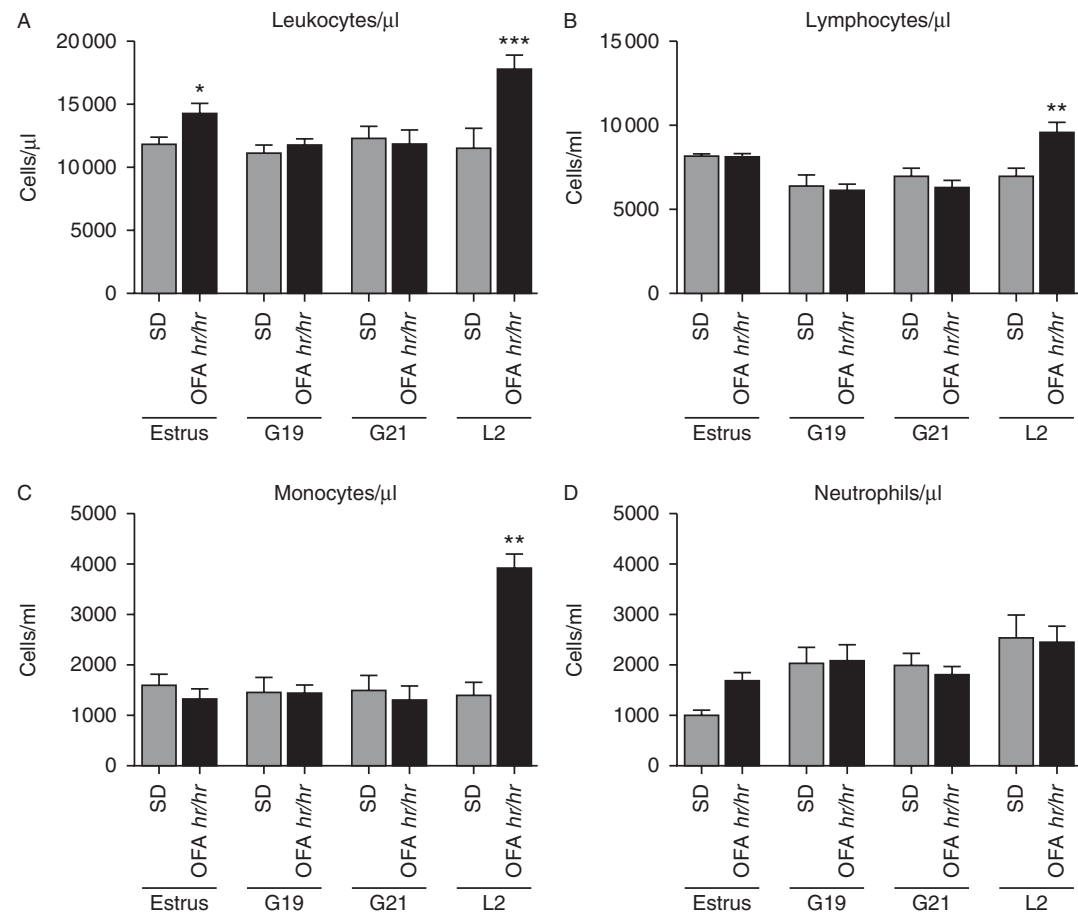

Figure 2 Peripheral white blood cell content in virgin (estrus), end of pregnancy (G19 and G21), and early lactation (L2) from SD and OFA $h r / h r$ rats. (A) Total leukocytes. (B) Lymphocytes. (C) Monocytes. (D) Neutrophils. Blood samples were collected at 0800-0830 h. Cells were counted using a hematology analyzer (ADVIA 120, Bayer). Values represent the mean \pm s.E.M. ${ }^{*} P<0.05$; ${ }^{* *} P<0.01$; ${ }^{* * *} P<0.001$; unpaired $t$-test; $S D$ vs OFA $h r / h r$ rats at the same reproductive state.

To further corroborate these results, we evaluated CD3 + $\mathrm{T}$ cells and CD45RA/B + B cells during mid-lactation. We found that on day 12 post partum, OFA $h r / h r$ rats present amounts of total leukocytes as well as CD3 + $T$ cells and CD45RA/B $+B$ cells that were similar to those presented by SD rats (Fig. 3E, F, G, H, and I). These results show that rats with deficient lactation have increased circulating $T$ and $B$ lymphocytes during early lactation, suggesting that PRL may be associated with Tand B-cell mobilization/migration from peripheral blood

to target tissues, most probably to MG at this stage of early lactation.

\section{PRL-R expression in peripheral blood leukocytes}

It is known that PRL-R is expressed in different cell types, including leukocytes, suggesting a direct modulation by PRL. We next studied the expression of PRL-R on peripheral blood leukocytes by flow cytometry on L2. As can be seen in Fig. 4A and B, OFA $h r / h r$ rats showed
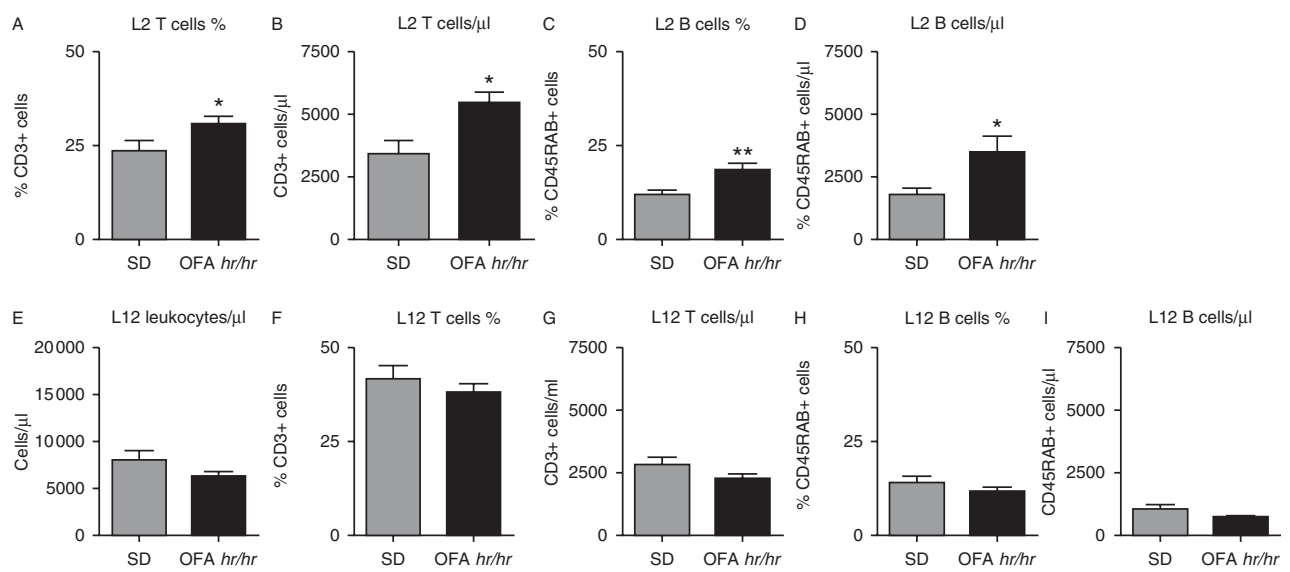

Figure $3 \mathrm{~B}$ - and T-cell populations in peripheral blood during early and mid-lactation. Flow cytometry analysis of peripheral blood leukocyte populations of SD and OFA $h r / h r$ rats during early (L2) and mid-lactation (L12). Peripheral blood samples were obtained from the tail vein. Total blood leukocytes were counted by a hematology analyzer (ADVIA 120, Bayer) or in a Neubauer chamber with Turk's solution to obtain absolute numbers. Red blood cells were eliminated by lysis buffer and leucocytes stained with specific anti-CD3-FITC and anti-CD45RA/B-FITC MABs following standard protocols and acquired in a FACS Calibur cytometer. \%, proportions from total leukocytes; absolute numbers represent total cell counts per $\mu$ l of whole blood. (A and B) \% and total CD3 + T cells in blood on L2. (C and D) $\%$ and total CD45RA/B + B cells in blood on L2. (E) Number of total leucocytes on L12. (F and G) \% and total CD3 + T cells in blood on L12. ( $\mathrm{H}$ and I) \% and total CD45RA/B + B cells in blood on L2. SD $(n=5-8)$ and OFA $h r / h r(n=5-8)$ rats. Values represent the mean \pm s.E.M. ${ }^{*} P<0.05 ;{ }^{* *} P<0.01$; unpaired $t$-test; SD vs OFA $h r / h r$ rats. 
A

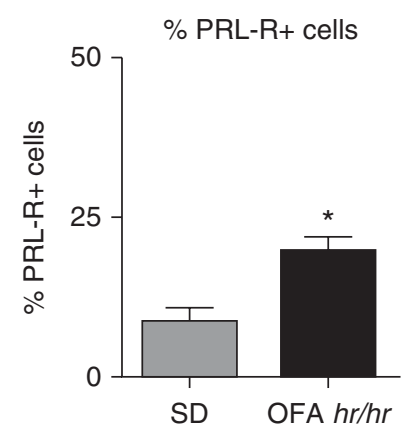

B

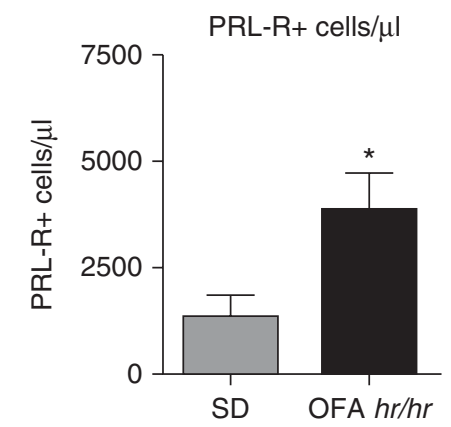

C

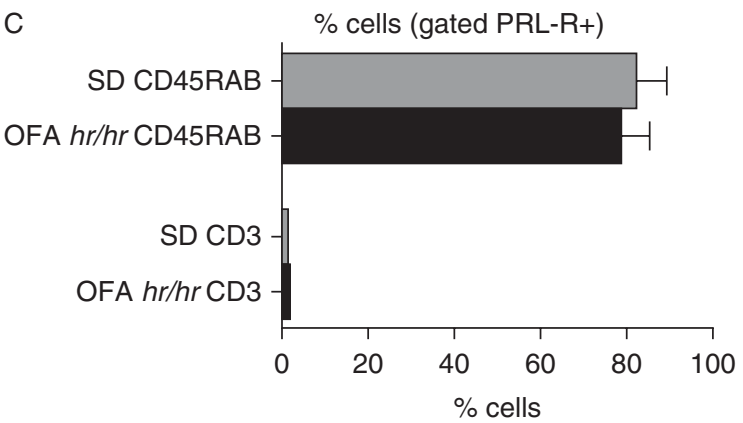

Figure 4 PRL-R + cell populations in peripheral blood during early lactation. Flow cytometry analysis of peripheral blood leukocyte populations of SD and OFA $h r / h r$ rats during early lactation (L2). Peripheral blood samples were obtained from the tail vein. Cells were stained with specific anti-CD3-FITC, anti-CD45RA/B-FITC, and purified anti-rat PRL Receptor clone U5 MABs with secondary PE-conjugated antibody following standard protocols and acquired in a FACS Calibur cytometer. \%, proportions from total leukocytes; absolute numbers represent total cell counts per $\mu \mathrm{l}$ of whole blood. (A and B) $\%$ and total PRL-R + cells in blood. (C) \% of CD45RA/B + B cells and CD3 + T cells of gated PRL-R + cells in blood. SD $(n=5)$ and OFA $h r / h r$ $(n=5)$ rats. Values represent the mean \pm s.E.M. ${ }^{*} P<0.05$; unpaired $t$-test; SD vs OFA $h r / h r$ rats.

increased number of circulating PRL-R + leukocytes compared with SD rats. PRL-R + cell population from both strains of rats was represented mainly by $B$ cells (Fig. 4C), and there were no differences in this proportion between the strains. Only $1-3 \%$ of PRL-R + cells were $\mathrm{CD} 3+\mathrm{T}$ cells. These results suggest that in a low serum PRL condition (hypoprolactinemia), PRL-R-expressing cells, potentially susceptible to PRL modulation, may accumulate in peripheral blood as a consequence of an impaired homing to target tissues, including MG.

\section{$B$ and $T$ cells in MG}

It is well known that resident lymphoid cell numbers increase in the MG during lactation in correlation with lobulo-alveolar epithelial cell expansion and differentiation (Parmely \& Manning 1983, Tanneau et al. 1999). To evaluate whether PRL levels may impact on the presence of $\mathrm{T}$ and $\mathrm{B}$ cells in $\mathrm{MG}$, we determined the number of mammary leukocytes in OFA $h r / h r$ and SD rats by FACS analysis. In inverse relation with the results observed in blood, mammary CD3 + T-cell population was decreased in OFA rats compared with SD rats (Fig. 5A, B and C). According to the results reported by Tanneau et al. (1999), we found a low percentage of CD45RA/B + cells in both strains, associated with no significant differences between both strains (Fig. 5D). However, when B cells differentiate to activated plasma cells, CD45RA/B expression decreases (Tanneau et al. 1999), which may account for the low numbers observed and the lack of differences between strains. Although monocytes were elevated in peripheral blood on L2, the presence of monocyte/granulocyte CD11b + cells on MG showed no differences between OFA $h r / h r$ and SD rats (Fig. 5E). To evaluate whether this observation, of a T-cell decrease in MG, persists over time, we evaluated the same populations studied during early lactation at 12 days post partum. We found that during midlactation, OFA $\mathrm{hr} / \mathrm{hr}$ rats continue presenting a decreased proportion of $\mathrm{CD} 3+\mathrm{T}$ cells and similar proportion of CD45RA/B and CD11b + cells than SD rats (Fig. 5J, $\mathrm{K}$, and $\mathrm{L}$ ).

To further evaluate T-cell homing events in our lactation-deficient model, we studied the specific location of CD3 + cells in MG by immunohistochemistry. As can be seen in Fig. 5F and G, we found that T-cell populations reside mainly in the stroma showing no differences between OFA $h r / h r$ and SD rats.

To analyze whether the decrease in T-cell population in MG was tissue specific, we also studied T-cell population in $\mathrm{CL}$, another PRL-responsive gland. As can be seen in Fig. $5 \mathrm{H}$ and I, CD3 + T-cell and CD11b+-cell populations were similar in CL of OFA and SD groups. We found only vestigial amounts of CD45RA/B + cells in CL from both strains. These results suggest that OFA $h r / h r$ and SD rats modulate T-cell homing events in a tissue-specific manner during early lactation.

\section{Chemokine receptor expression during early lactation}

Chemokine receptors are expressed in many cell types such as leukocytes, epithelial, endothelial, and fibroblasts. These receptors are involved in migration/homing, inflammation, angiogenesis, and tissue development. Thus, differences in the expression of chemokine receptors may help to understand the differences found in circulating and mammary 

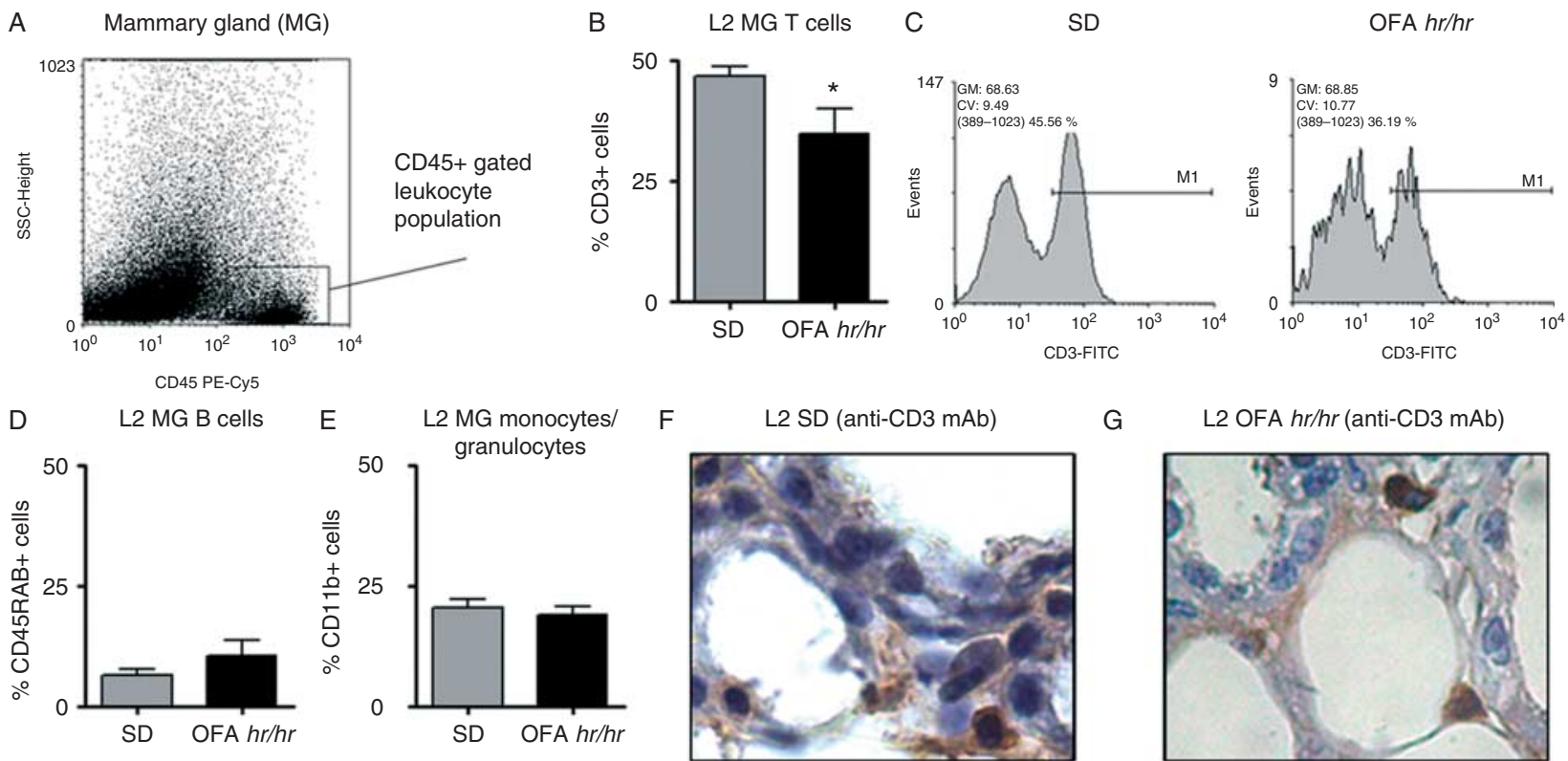

G

L2 OFA $h r / h r($ anti-CD3 mAb)
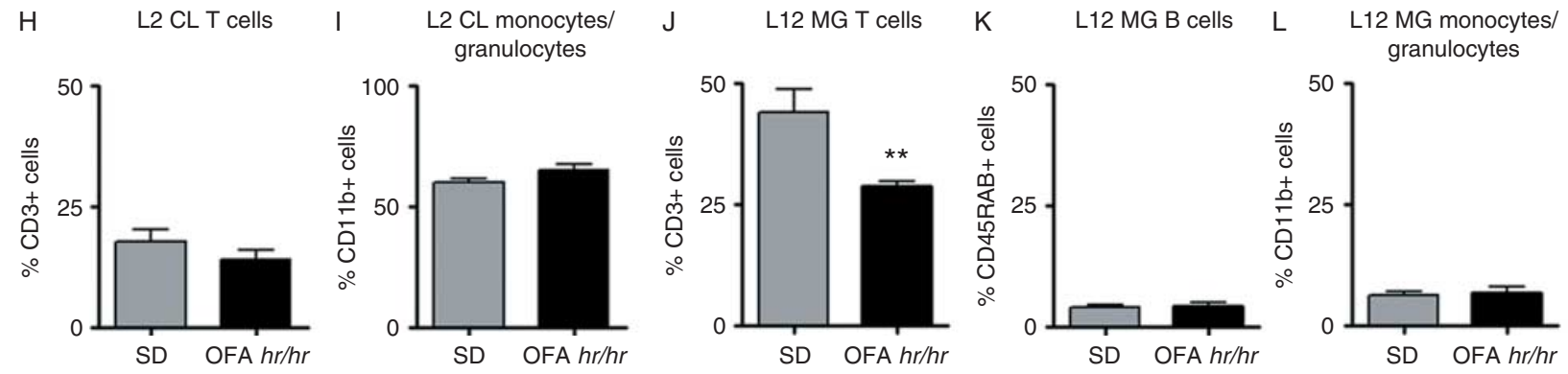

Figure 5 T-cell population in mammary gland $(\mathrm{MG})$ and corpus luteum $(\mathrm{CL})$ during early lactation and in MG during mid-lactation. Inguinal $\mathrm{MG}$ and $\mathrm{CL}$ were rapidly dissected, minced, and subjected to enzymatic digestion for $1 \mathrm{~h}$ as described in the Materials and Methods section. Cells were dispersed and washed through a $200 \mu \mathrm{m}$ nylon cell strainer. Resident leukocyte composition from MG and CL was evaluated by FACS using anti-rat CD45 PE-Cy5, anti-rat CD3-FITC, anti-rat CD45RA/B-FITC, and anti-rat CD11b-PE MABs. (A) Representative gating of total leukocytes with CD45 PE-Cy5 vs SCC dot plots. (B and C) \% of CD3 + T cells and representative histograms of CD3 staining from MG on L2 (MG CD45+ gated cells). (D and E) CD45RA/B + B cells and CD11b + monocyte/granulocyte cells from MG on L2. (J K, and L) \% of CD3 + T cells, CD45RA/B + B cells, and $\mathrm{CD} 11 \mathrm{~b}+$ monocyte/granulocyte cells from MG on L12. Location of CD3 + T cells in MG tissue on L2 was assayed by immunohistochemistry with CD3 staining of MG from SD (F) and OFA $h r / h r(G)$ rats. 400×. (H and I) Flow cytometry analysis of CD3 + T cells and CD11b+ cells from CL on L2. $\mathrm{SD}(n=5)$ and OFA $h r / h r(n=5)$ rats. Values represent the mean \pm s.E.M. $* P<0.05 ; * *<0.01$; unpaired $t$-test; SD vs OFA $h r / h r$ rats.

lymphocytes between SD and OFA $h r / h r$ rats. With this aim, we studied the expression of chemokine receptors reported to be regulated by PRL, such as CCR1, CCR2, CCR7, CXCR3, and CXCR5, in peripheral blood T and B cells in SD and OFA $h r / h r$ rats during early lactation. These chemokine receptors have multiple ligands conferring pleiotropic functions (CCR1: CCL3, 5, 7, 8, 13, 14, 15, 16, and 23; CCR2: CCL2, 7, 8, 12, and 13; CCR7: CCL19, 21; CXCR3: CXCL9, 10, 11; and CXCR5: CXCL13) (Proudfoot 2002). As can be seen in Fig. 6A and $B$, peripheral blood $T$ and $B$ cells of OFA and SD rats showed no major differences in the expression of CCR1, CCR2, CCR7, CXCR3, and CXCR5. To further describe homing events and tissue homeostasis under low PRL levels, we also measured mRNA expression of chemokine receptors on mammary tissue (Fig. 6C). There were no significant differences in MG expression of CCR1, CCR2, CCR5, CCR7, CXCR3, and CXCR5, although CCR7, CXCR3, and CXCR5 showed a tendency to be higher in OFA rats. In contrast, CCR3 mRNA expression was increased in OFA $h r / h r$ rats compared with SD rats. As CCR3 is mainly associated with proinflammatory events and tissue repair, we speculate that its upregulation may be due to remodeling signals secondary to the hypoprolactinemia that result in deficient gland differentiation and perhaps in early induction of involution. We also studied the expression of PRL-R in total MG tissue of OFA $h r / h r$ and SD rats to estimate the mammary responsiveness to PRL in both strains. As can be seen in Fig. 6C, there were no differences in PRL-R mRNA expression in OFA $h r / h r$ rats compared with SD rats, suggesting a similar capacity to respond to PRL in both strains. 


\section{Discussion}

Despite major advances in the understanding of the interplay between hormones and the immune system, the specific role of PRL during pregnancy and lactation in

A CD3+ T cells

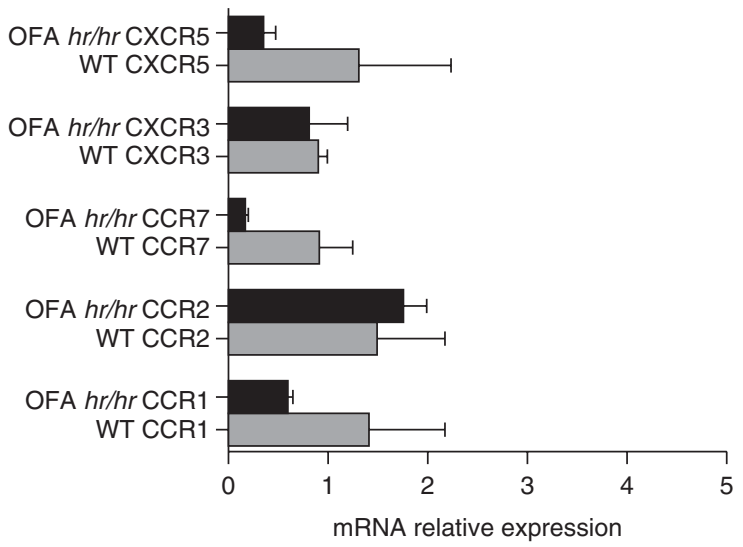

B

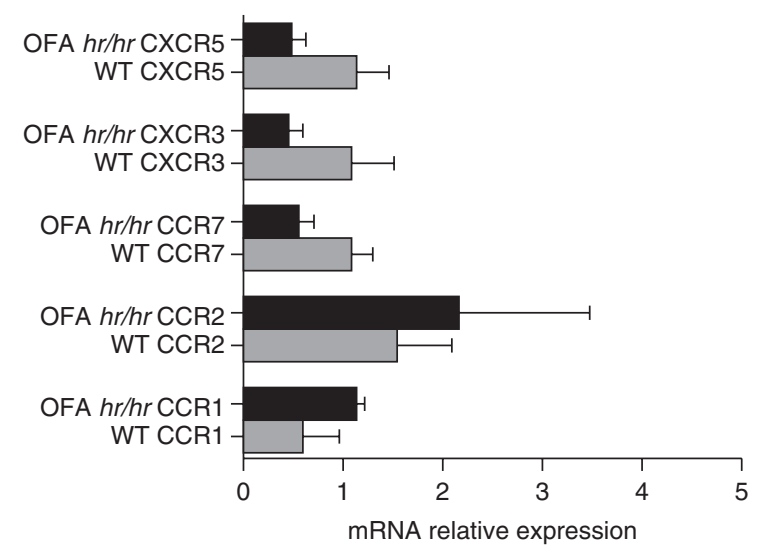

C

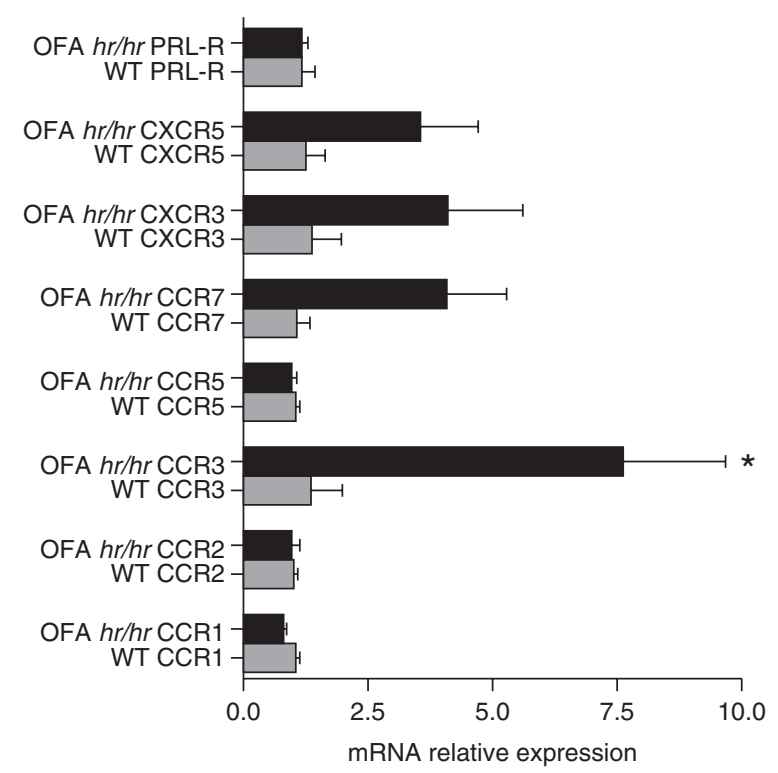

lymphoid cell function remains to be elucidated. In this study, we show that the proportion of circulating T cells on early lactating hypoprolactinemic OFA $h r / h r$ rats is higher than that in normoprolactinemic SD rats. Furthermore, in the OFA $h r / h r$ rats, resident MG T cells show a decreased proportion compared with SD rats.

A few studies have described that lactation hormonedriven signals induce T-cell mobilization from mucosaassociated lymphoid tissue and lymph nodes, and that elevated PRL levels direct these cells to mammary tissue (Roux et al. 1977, Weisz-Carrington et al. 1977). Our observation that T-cell proportion was increased in blood and decreased in MG concomitantly with low PRL levels in OFA $h r / h r$ rats during early lactation (but not during mid-lactation) suggests that in the presence of hypoprolactinemia mobilized $T$ cells from lymphoid tissues during lactation cannot reach the $M G$, accumulating in blood. These results suggest that OFA $h r / h r$ rats show impaired T-cell mobilization, homing, or migration. This event seems to be organ specific, as there were no differences between strains in luteal T-cell abundance. Although some reports have identified PRL-R on immune cells as well as a direct regulation of PRL over T cells (LaVoie \& Witorsch 1995, Yu-Lee et al. 1998, Buckley 2001, Montgomery 2001, Urtishak et al. 2001), we only detected PRL-R in a very low proportion of circulating T cells. Despite not having detected PRL-R by FACS on the expected proportion of the T-cell population, we could not exclude that a very low expression (below the detection threshold of the technique) of PRL-R is able to make T cells sensitive to PRL. These results lead us to present two probable mechanisms: i) a direct effect of PRL-modulating T-cell recruitment such as inducing specific adhesion molecules in the presence of PRL, and ii) an indirect modulation by circulating PRL through induction of T-cell-recruiting chemokines by MG epithelial cells. The fact that we found PRL-R in a very low proportion of $\mathrm{T}$ cells mostly supports the second hypothesis. To support this notion, it has been reported that PRL induces expression of T-cell chemokines such as CCL20, CXCL9, CXCL10, and CXCL11 in epithelial cells (keratinocytes) (Kanda \& Watanabe 2007, Kanda et al. 2009). Also, mammary epithelial cells can express T-cell chemokines such as RANTES and IL8 in response to cytokines (Michie et al. 1998). Considering all this data, it is likely that PRL plays a role, direct or indirect, in

Figure 6 Chemokine receptor expression during early lactation. B- and T-cell populations from peripheral blood were purified by magnetic cell sorting as described in the Materials and Methods section. Total RNA from T and B cell and MG was purified using TRIzol reagent. Firststrand CDNAs were made by reverse transcriptase standard protocol. CDNA measurements were performed by real-time PCR with Eva Green detection. (A and B) Relative expression of chemokine receptors from peripheral blood $\mathrm{CD} 3+$ and $\mathrm{CD} 45 \mathrm{RA} / \mathrm{B}+$ cells respectively. (C) Relative expression of chemokine receptors from MG. SD $(n=4)$ and OFA $h r / h r(n=4)$ rats. Values represent the mean \pm S.E.M. ${ }^{*} P<0.05$; unpaired $t$-test; SD vs OFA $h r / h r$ rats. 
T-cell homing to MG during early lactation. In addition, the fact that OFA $h r / h r$ rats also showed a decrease in T-cell proportion in MG during mid-lactation without changes in peripheral blood compared with SD rats could be due to events observed during early lactation. This result further reinforces the importance of this work in which a subnormal condition during early lactation can last over time. Here, we provide for the first time an association between PRL levels and T-cell population in $M G$ in a physiological context. We can speculate that the hyperprolactinemia of early lactation contributes to the maintenance of $T$ cells in MG.

Although B cells, characterized here as CD45RA/B + cells, accumulated in blood, perhaps following the same mechanism proposed for $\mathrm{T}$ cells, we did not observe changes in MG B-cell population. These results may be related to the lack of expression of CD45RA/B on antibodyproducing cells, such as plasma cells. B cells locate to MG mainly to differentiate into plasma cells to produce antibodies, losing CD45RA/B expression (Tanneau et al. 1999). Tanneau et al. (1999) found that MG B cells in mice (CD19+ and CD45R/B220+) represent a small population that does not vary during pregnancy or lactation. Further studies need to be done to evaluate the precise role of PRL in plasma cell populations present in MG.

We observed that a decrease in the number of MG CD3 + T cells occurs when PRL levels are low, suggesting mammary homing modulation by this hormone. These events may occur mainly as a dynamic equilibrium of cells migrating to the MG rather than an accumulation during pregnancy and lactation involving several molecules such as integrins, addressins, chemokines, and chemokine receptors. The approach we made to address this issue was to evaluate the expression of chemokine receptors on purified T cells in blood. Unfortunately, we could not shed light on the mechanism responsible for the suggested PRL modulation of T-cell homing to MG as no major changes in the mRNA expression of CCR1, CCR2, CCR7, CXCR3, and CXCR5 chemokine receptors were found. To support this notion, reports indicate that at least two adhesion molecules, MadCam and $\alpha 4 \beta 7$, are involved in MG homing during pregnancy and lactation (Tanneau et al. 1999, van der Feltz et al. 2001, Nishimura 2003, Bourges et al. 2008). The possibility cannot be excluded that additional changes may occur during T-cell recruitment and maintenance in MG, involving addressins, integrins, and chemokine receptors' expression on T cells as well as chemokine production by mammary epithelial cells under hormone/cytokine stimulation. The observation of an increase in CCR3 expression in MG can be associated with derived endothelial and epithelial cells remodeling signals rather than a leukocyte-driven function. We speculate that CCR3 upregulation may be due to remodeling signals secondary to hypoprolactinemia that produce an altered MG differentiation and early involution. As PRL regulates diverse physiological functions such as proliferation, differentiation, and cell survival, it is more probable that the mechanism responsible for the proposed PRL modulation of T-cell homing to MG involves a complex network between MG (epithelial/endothelial cells) and mobilized T cells (from lymphoid tissues) than a simple induction of adhesion molecule expression.

As PRL is the most important hormone in MG development during lactation, we propose that this hormone may help to modulate lymphocyte homing to MG that may be coordinated mainly by addressins, integrins, chemokines, and chemokine receptors. Our suggested working model is that PRL in the early lactation phase induces MG cells (endothelial/epithelial/stromal) to secrete T-cell chemoattractants in order to recruit them from peripheral blood. The most important implications of our work are that PRL could be involved in diverse T-cell migration and homing events, resulting in a detrimental role in T cells under hyper- as well as hypoprolactinemic conditions such as autoimmune and hormonal imbalances. The mechanism and the immune mediator/s by which PRL may modulate T-cell migration and homing to MG still remain to be elucidated. In addition, further studies are needed to test whether T-cell mobilization from lymphoid tissue is (or is not) affected in situations of low circulating PRL levels.

In conclusion, in this study, we show that hypoprolactinemia during early lactation is accompanied with altered T-cell distribution, which is elevated in circulation and decreased in mammary tissue. These results suggest that during lactation, PRL contributes in part to modulation of T-cell homing to MG. These events would help in the understanding of PRL-associated immune disease and should be considered in future studies.

\section{Declaration of interest}

The authors declare that there is no conflict of interest that could be perceived as prejudicing the impartiality of the research reported.

\section{Funding}

This work was supported by grants from the Agencia Nacional de Promoción Científica y Tecnológica (FONCYT) and from the Consejo Nacional de Investigaciones Científicas y Técnicas de Argentina (CONICET). J P Mackern-Oberti is a post-doctoral fellow from CONICET. G A Jahn, L M Vargas-Roig and S R Valdez are Career Research members of CONICET.

\section{Acknowledgements}

The authors thank Norma Carreño for her skillful technical assistance. They also thank Dr Adriana Gonzalez and Dr Carolina Marin for their helpful assistance with FACS analysis; Dr Isabel A Gimenez for her assistance with the hematology analyzer; and Dr Virginia E Rivero, Dr Jean Djiane, and Dr Paul Kelly for providing MABs. 


\section{References}

Bazzi H, Kljuic A, Christiano AM \& Panteleyev AA 2004 Intragenic deletion in the desmoglein 4 gene underlies the skin phenotype in the Iffa Credo "hairless" rat. Differentiation 72 450-464. (doi:10.1111/j.1432-0436. 2004.07208010.x)

Bouchard B, Ormandy CJ, Di Santo JP \& Kelly PA 1999 Immune system development and function in prolactin receptor-deficient mice. Journal of Immunology 163 576-582.

Bourges D, Meurens F, Berri M, Chevaleyre C, Zanello G, Levast B, Melo S, Gerdts V \& Salmon H 2008 New insights into the dual recruitment of $\operatorname{lgA}+\mathrm{B}$ cells in the developing mammary gland. Molecular Immunology 45 3354-3362. (doi:10.1016/j.molimm.2008.04.017)

Buckley AR 2001 Prolactin, a lymphocyte growth and survival factor. Lupus 10 684-690. (doi:10.1191/096120301717164912)

Chabaudie N, Le Jan C, Olivier M \& Salmon H 1993 Lymphocyte subsets in the mammary gland of sows. Research in Veterinary Science 55 351-355. (doi:10.1016/0034-5288(93)90106-P)

Dimitrov S, Lange T, Fehm HL \& Born J 2004 A regulatory role of prolactin, growth hormone, and corticosteroids for human T-cell production of cytokines. Brain, Behavior, and Immunity 18 368-374. (doi:10.1016/j. bbi.2003.09.014)

Dogusan Z, Hooghe R, Verdood P \& Hooghe-Peters EL 2001 Cytokine-like effects of prolactin in human mononuclear and polymorphonuclear leukocytes. Journal of Neuroimmunology 120 58-66. (doi:10.1016/ S0165-5728(01)00420-9)

Elbourne KB, Keisler D \& McMurray RW 1998 Differential effects of estrogen and prolactin on autoimmune disease in the NZB/NZW F1 mouse model of systemic lupus erythematosus. Lupus 7 420-427. (doi:10.1191/096120398678920352)

van der Feltz MJ, de Groot N, Bayley JP, Lee SH, Verbeet MP \& de Boer HA 2001 Lymphocyte homing and Ig secretion in the murine mammary gland. Scandinavian Journal of Immunology 54 292-300. (doi:10.1046/j. 1365-3083.2001.00933.x)

Gerlo S, Verdood P, Hooghe-Peters EL \& Kooijman R 2005 Modulation of prolactin expression in human T lymphocytes by cytokines. Journal of Neuroimmunology 162 190-193. (doi:10.1016/j.jneuroim.2005.02.008)

Holmes K, Lantz LM, Fowlkes BJ, Schmid I \& Giorgi JV 2001 Preparation of cells and reagents for flow cytometry. In Current Protocols in Immunology, ch 5, Unit 5.3. John Wiley \& Sons, Inc. (doi:10.1002/ 0471142735.im0503s44)

Horseman ND, Zhao W, Montecino-Rodriguez E, Tanaka M, Nakashima K, Engle SJ, Smith F, Markoff E \& Dorshkind K 1997 Defective mammopoiesis, but normal hematopoiesis, in mice with a targeted disruption of the prolactin gene. $E M B O$ Journal $\mathbf{1 6}$ 6926-6935. (doi:10.1093/emboj/16.23.6926)

Kanda N \& Watanabe S 2007 Prolactin enhances Interferon- $\gamma$-induced production of CXC ligand 9 (CXCL9), CXCL10, and CXCL11 in human keratinocytes. Endocrinology 148 2317-2325. (doi:10.1210/en.2006-1639)

Kanda N, Shibata S, Tada Y, Nashiro K, Tamaki K \& Watanabe S 2009 Prolactin enhances basal and IL-17-induced CCL20 production by human keratinocytes. European Journal of Immunology 39 996-1006. (doi:10.1002/eji.200838852)

Kooijman R, Rijkers GT \& Zegers BJ 1996 IGF-I potentiates interleukin-2 production in human peripheral T cells. Journal of Endocrinology 149 351-356. (doi:10.1677/joe.0.1490351)

Kunkel EJ \& Butcher EC 2002 Chemokines and the tissue-specific migration of lymphocytes. Immunity 16 1-4. (doi:10.1016/S10747613(01)00261-8)

LaVoie HA \& Witorsch RJ 1995 Investigation of intracellular signals mediating the anti-apoptotic action of prolactin in $\mathrm{Nb} 2$ lymphoma cells. Proceedings of the Society for Experimental Biology and Medicine 209 257-269. (doi:10.3181/00379727-209-43901)

Marcondes FK, Bianchi FJ \& Tanno P 2002 Determination of the estrous cycle phases of rats: some helpful considerations. Brazilian Journal of Biology 62 609-614. (doi:10.1590/S1519-69842002000400008)

Matera L, Mori M \& Galetto A 2001 Effect of prolactin on the antigen presenting function of monocyte-derived dendritic cells. Lupus $\mathbf{1 0}$ 728-734. (doi:10.1191/096120301717164967)

Michie C, Tantscher E, Schall T \& Rot A 1998 Physiological secretion of chemokines in human breast milk. European Cytokine Network 9 123-129.
Montes de Oca P, Macotela Y, Nava G, Lopez-Barrera F, de la Escalera GM \& Clapp C 2005 Prolactin stimulates integrin-mediated adhesion of circulating mononuclear cells to endothelial cells. Laboratory Investigation 85 633-642. (doi:10.1038/labinvest.3700256)

Montgomery DW 2001 Prolactin production by immune cells. Lupus 10 665-675. (doi:10.1191/096120301717164895)

Morikawa K, Oseko F \& Morikawa S 1993 Immunosuppressive property of bromocriptine on human B lymphocyte function in vitro. Clinical and Experimental Immunology 93 200-205. (doi:10.1111/j.1365-2249. 1993.tb07966.x)

Morikawa K, Oseko F \& Morikawa S 1994 Immunosuppressive activity of bromocriptine on human T lymphocyte function in vitro. Clinical and Experimental Immunology 95 514-518. (doi:10.1111/j.1365-2249. 1994.tb07028.x)

Nagafuchi H, Suzuki N, Kaneko A, Asai T \& Sakane T 1999 Prolactin locally produced by synovium infiltrating $\mathrm{T}$ lymphocytes induces excessive synovial cell functions in patients with rheumatoid arthritis. Journal of Rheumatology 26 1890-1900.

Nishimura T 2003 Expression of potential lymphocyte trafficking mediator molecules. Veterinary Research 34 3-10. (doi:10.1051/vetres:2002045)

Parmely MJ \& Manning LS 1983 Cellular determinants of mammary cellmediated immunity in the rat: kinetics of lymphocyte subset accumulation in the rat mammary gland during pregnancy and lactation. Annals of the New York Academy of Sciences 409 517-533. (doi:10.1111/j. 1749-6632.1983.tb26896.x)

Peeva E, Venkatesh J, Michael D \& Diamond B 2004 Prolactin as a modulator of B cell function: implications for SLE. Biomedicine \& Pharmacotherapy 58 310-319. (doi:10.1016/j.biopha.2004.04.007)

Proudfoot AEI 2002 Chemokine receptors: multifaceted therapeutic targets. Nature Reviews. Immunology 2 106-115. (doi:10.1038/nri722)

Roux ME, McWilliams M, Phillips-Quagliata JM, Weisz-Carrington P \& Lamm ME 1977 Origin of IgA-secreting plasma cells in the mammary gland. Journal of Experimental Medicine 146 1311-1322. (doi:10.1084/ jem.146.5.1311)

Tanneau GM, Hibrand-Saint Oyant L, Chevaleyre CC \& Salmon HP 1999 Differential recruitment of T- and IgA B-lymphocytes in the developing mammary gland in relation to homing receptors and vascular addressins. Journal of Histochemistry and Cytochemistry $\mathbf{4 7}$ 1581-1592. (doi:10.1177/002215549904701210)

Urtishak SL, Mckenna EA \& Mastro AM 2001 Prolactin and prolactin receptor expression in rat small intestine intraepithelial lymphocytes during neonatal development. Developmental Immunology 8 319-330. (doi:10.1155/2001/10175)

Valdez SR, Penissi AB, Deis RP \& Jahn GA 2007 Hormonal profile and reproductive performance in lactation deficient (OFA hr/hr) and normal (Sprague-Dawley) female rats. Reproduction 133 827-840. (doi:10.1530/REP-06-0032)

Valdez SR, Bonafede MM, Carreño NB, Deis RP \& Jahn GA 2012 Differential sensitivity to stress in rats of OFA hr/hr strain may contribute to their lactation failure. Comparison with Wistar and Sprague Dawley strains. Stress 15 361-377. (doi:10.3109/10253890.2011.624223)

Watson CJ, Oliver CH \& Khaled SD 2011 Cytokine signaling in mammary gland development. Journal of Reproductive Immunology 88 124-129. (doi:10.1016/j.jri.2010.11.006)

Weisz-Carrington P, Roux ME \& Lamm ME 1977 Plasma cells and epithelial immunoglobulins in the mouse mammary gland during pregnancy and lactation. Journal of Immunology 119 1306-1307.

Yasugi T, Kaido T \& Uehara Y 1989 Changes in density and architecture of microvessels of the rat mammary gland during pregnancy and lactation. Archives of Histology and Cytology 52 115-122. (doi:10.1679/aohc.52.115)

Yu-Lee L, Luo G, Moutoussamy S \& Finidori J 1998 Prolactin and growth hormone signal transduction in lymphohaemopoietic cells. Cellular and Molecular Life Sciences 54 1067-1075. (doi:10.1007/s000180050235)

Received 1 October 2012

First decision 5 November 2012

Revised manuscript received 20 June 2013

Accepted 26 June 2013 